\title{
Immunogenic response of Brucella canis virB10 and virB11 mutants in a murine model
}

\author{
E. Palomares-Resendiz ${ }^{1}$, B. Arellano-Reynoso² , R. Hernández-Castro ${ }^{3}$, V. Tenorio-Gutiérrez ${ }^{1}$, E. Salas-Téllez ${ }^{4}$, \\ F. Suárez-Güemes ${ }^{2}$ and Efrén Díaz-Aparicio ${ }^{1 *}$ \\ ' CENID Microbiología, Instituto Nacional de Investigaciones Forestales, Agrícolas y Pecuarias, Mexico City, México \\ ${ }^{2}$ Departamento de Microbiología e Inmunología, Facultad de Medicina Veterinaria y Zootecnia, Universidad Nacional Autónoma de México, Mexico City, México \\ ${ }^{3}$ Departamento de Ecología de Agentes Patógenos, Hospital General "Dr. Manuel Gea González," Secretaría de Salud, Mexico City, México \\ ${ }^{4}$ Facultad de Estudios Superiores Cuautitlán, Universidad Nacional Autónoma de México, Mexico City, México
}

Edited by:

Thomas A. Ficht, Texas A\&M

University, USA

Reviewed by:

David O'Callaghan, INSERM, France

Chad J. Roy, Tulane University, USA

${ }^{*}$ Correspondence:

Efrén Díaz-Aparicio, Centro Nacional de Investigaciones Disciplinarias en

Microbiología Animal, Instituto

Nacional de Investigaciones

Forestales, Agrícolas y Pecuarias,

Carretera Federal México-Toluca Km.

15.5, Cuajimalpa, Mexico City, Distrito

Federal 05110, México.

e-mail: efredia@yahoo.com
The virB locus, which encodes the type IV secretion system, is a major component of virulence in Brucella. A non-polar virB10 mutant and a virB11 deletion mutant were constructed in Brucella canis. In the mouse model, both mutants were cleared at day 21 post-infection, indicating reduced virulence in mice. After challenging with wild-type $B$. canis, the amounts of CFU recovered at day 15 were significantly lower in the group previously vaccinated with the virB10 mutant. Levels of $\lg G 1, \lg G 2 a$, IgG2b, and IgM, the induction of the cytokines IL2 , IL-4, IL-10, and the production of IFN- $\gamma$ were measured in lymphocyte cultures. All strains elicited similar levels of different antibody isotype profiles, and no significant differences were detected $(P<0.05)$. The wild-type strain induced a rapid and strong INF- $\gamma$ response at $24 \mathrm{~h}$, while both mutants induced mild INF- $\gamma$ responses at $24 \mathrm{~h}$, which remained constant over the course of sampling. Our results suggest that the virB mutants elicit a protective immunity and may be considered as candidates for studies to be conducted in dogs against canine brucellosis.

Keywords: Brucella canis, virB mutants, mouse model

\section{INTRODUCTION}

The Brucella genus consists of Gram-negative, facultative intracellular microorganisms that cause disease in diverse animal species, including humans (Sieira et al., 2000). Brucella species can survive within professional and non-professional phagocytes, thus avoiding the host defense mechanisms. Host resistance to facultative intracellular pathogens such as Brucella depends mainly on the induction of cell-mediated immunity (Golding et al., 2001; Baldwin and Parent, 2002; Baldwin and Goenka, 2006). The capacity of antigens to selectively induce a Th1 or Th2 response is an important aspect in the development of vaccines against intracellular Brucella pathogens (Zhan et al., 1996). Studies performed in the murine model show that live attenuated vaccines induce a cellular response characterized by the production of IFN- $\gamma$ and IL-2 (Zhan et al., 1995). In the case of canine brucellosis, no vaccine is available; thus, studies concerning Brucella canis virulence factors, as well as interactions between host-pathogen should be performed in order to develop immunogens to prevent disease in dogs.

A virulence factor that has been shown to be essential for $\mathrm{Bru}$ cella is the type IV secretion system (T4SS), which is encoded by the virB operon (Hong et al., 2000; Sieira et al., 2000) and is required for the establishment and persistence of infection in the murine model (Sun et al., 2002; Rolan and Tsolis, 2008). It has been shown that virB mutants of B. abortus and B. melitensis have reduced capacities to survive and replicate in professional and non-professional phagocytic cells (Sun et al., 2005; Rolan and Tsolis, 2008).
In this study, a $B$. canis non-polar virB10 mutant and a virB11 null mutant were evaluated for their roles in protecting against an experimental challenge. The induction of the cytokines IL-2, IL-4, and IL-10 as well as the production of IFN- $\gamma$ in spleen cells from mice inoculated with $B$. canis mutants were assessed, as was the production of IgG1, IgG2a, IgG2b, and IgM in the murine model.

\section{MATERIALS AND METHODS BACTERIA STRAINS, MEDIA, AND CULTURE CONDITIONS}

The B. canis wild-type strain, the mutants virB10 and virB11, and their complemented mutants were cultured at $37^{\circ} \mathrm{C}$ in Brucella agar or Brucella broth with orbital shaking (200 rpm) for $18 \mathrm{~h}$. Escherichia coli JM109 and DH5 $\alpha$ strains were cultured at $37^{\circ} \mathrm{C}$ in Luria-Bertani agar or broth with orbital shaking $(200 \mathrm{rpm})$ for $18 \mathrm{~h}$. When necessary, the following antibiotics were added: gentamycin $(2.5 \mu \mathrm{g} / \mathrm{ml})$ and ampicillin $(50 \mu \mathrm{g} / \mathrm{ml}$; SIGMA Aldrich, St. Louis, MO, USA).

\section{CONSTRUCTION OF virB10 AND virB11 MUTANTS}

To obtain a non-polar mutation in the virB 10 gene, an insertional mutant was constructed. The plasmid pB2A3, which contains the virB10 gene, was linearized with $\mathrm{NruI}$ and ligated with a $0.7-\mathrm{kb}$ SmaI fragment encoding a gentamycin resistance cassette. The resulting plasmid, pB2A3::Gm, was electroporated into B. canis where it is incapable of autonomous replication, and doublehomologous recombinant events $\left(\mathrm{Gm}^{\mathrm{r}} \mathrm{Amp} \mathrm{p}^{\mathrm{s}}\right)$ were selected using gentamicin resistance (Sieira et al., 2000). 
To generate a mutant with a deletion in the virB11 gene, a $1.2-\mathrm{kb}$ fragment of the plasmid pGEM-T-virB10 that contained a virB10 gene was ligated into pBluescript KSII to generate the plasmid pBlue-virB10. A 0.7-kb BamHI fragment encoding a gentamycin resistance cassette was ligated into the BamHI site of pBlue-virB10 to generate the plasmid pBlue-virB10-Gm. An 850 -bp that contained the orf13-orf12 region was cloned into the pGEM-T Easy vector and was recovered by Not I digestion. This fragment was ligated into pBlue-virB10 at the Not I site to generate the plasmid pBlue-virB10::Gm-ORF13-ORF12. Five micrograms of pBlue-virB10::Gm-ORF13-ORF12 was electroporated into wild-type $B$. canis. Accordingly, colonies that were $\mathrm{Gm}^{\mathrm{r}}$ and $A \mathrm{mp}^{\mathrm{s}}$ were selected as possible double-homologous recombinants.

\section{COMPLEMENTATION OF BRUCELLA CANIS virB10 AND virB11 NON-POLAR MUTANTS}

The entire virB10 gene was cloned into the pBBR1MCS-4 plasmid at the EcoRI site to generate the pGP10c plasmid. The pGP10c plasmid was electroporated into B. canis virB10::Gm, and $A \mathrm{mp}^{\mathrm{r}}$ strains were selected. The presence of the plasmid was confirmed by alkaline lysis. To restore the virB 11 gene, the complete gene was introduced into the pBBR1MCS-4 plasmid at the EcoRI site to generate the pGP11c plasmid. The pGP11c plasmid was then transformed into B. canis $\Delta$ virB11::Gm by electroporation; $A_{\mathrm{mp}}{ }^{\mathrm{r}}$ strains were selected, and the presence of the plasmid was confirmed by alkaline lysis.

\section{EXPERIMENTAL INFECTION IN MICE}

Female 6- to 7-week-old BALB/c mice were obtained from Harlan, Mexico. The mice were placed in cages with water and feed ad libitum and were acclimated for 1 week before beginning the experiments. Three groups of 16 mice each were formed for the in vivo virB mutant protection experiments.

\section{SURVIVAL OF virB MUTANTS IN MICE}

The survival of $\operatorname{vir} B$ mutants, of the complemented mutants virB10::Gm and $\Delta$ virB11::Gm and of wild-type B. canis was determined by quantitating the CFU of each strain in mouse spleens at different time intervals post-infection. Groups of 157 -weekold female BALB/c mice were injected i.p. with $1.4 \times 10^{8} \mathrm{CFU}$ of the virB10::Gm strain or the $\Delta$ virB11::Gm strain in $0.1 \mathrm{ml}$ of PBS. At 7, 21, 35, and 45 days post-infection (p.i.), animals were sacrificed, and spleens were removed and homogenized in PBS. Tissue homogenates were serially diluted with PBS and were plated onto tryptic soy broth-agar with the appropriate antibiotics to determine the numbers of CFU per spleen.

For protection experiments, groups of 16 mice were immunized i.p. with $1.4 \times 10^{8} \mathrm{CFU}$ of strain virB10::Gm or strain $\Delta$ virB11::Gm or with PBS (unvaccinated control). Six weeks postinoculation, both groups were challenged i.p. with $5 \times 10^{4} \mathrm{CFU}$ of virulent wild-type $B$. canis per mouse. Two and 4 weeks postchallenge, eight mice per group were sacrificed, and the numbers of CFU recovered from spleens were determined. Protection units were defined as the difference between the mean log of the numbers of CFU from immunized mice and that of the numbers of CFU from mice receiving saline.

\section{LYMPHOCYTE CULTURES AND CYTOKINE INDUCTION}

Three groups of five 6- to 7-week-old female BALB/c mice were immunized intraperitoneally with $1.4 \times 10^{8} \mathrm{CFU} / \mathrm{ml}$ of $B$. canis or the $\operatorname{vir} B$ mutants; each mouse was inoculated at both day 0 and 8. Fifteen days after the first inoculation, the mice were sacrificed, and spleens were extracted for lymphocyte cultures. Spleens from each group of mice were combined and washed three times with Hank's balanced salt solution and were placed in a Petri dish that contained $5 \mathrm{ml}$ of RPMI 1640 medium (Gibco, Laboratories) supplemented with $100 \mathrm{U} / \mathrm{ml}$ of penicillin and $100 \mu \mathrm{g} / \mathrm{ml}$ of streptomycin. The spleens were wrapped with sterile gauze to retain tissue portions and were then macerated. The cellular suspension was transferred into a tube with $5 \mathrm{ml}$ of the same medium and was centrifuged at $400 \times g$ for $3 \mathrm{~min}$. The cells were re-suspended in $0.17 \mathrm{M}$ ammonium chloride for $5 \mathrm{~min}$ at $4^{\circ} \mathrm{C}$ to lyse erythrocytes, and they were then washed three additional times with RPMI. Cells were re-suspended in RPMI medium enriched with $20 \%$ bovine fetal serum, $200 \mathrm{mM} \mathrm{L}$-glutamine and $0.1 \mathrm{mM}$ nonessential amino acids. A total of $6.5 \times 10^{6}$ mouse lymphocytes were stimulated with their corresponding mutant or the wild-type strain and were then each distributed into five wells in cell culture plates (Nunclon, Rochester, NY, USA). Wells were inoculated with $10^{8} \mathrm{CFU}$ of each one of the strains, and the plates were then incubated at $37^{\circ} \mathrm{C}$ with $5 \% \mathrm{CO}_{2}$. A positive control was inoculated with concanavalin A. Supernatants were collected at 24, 48, 72, 96, and $120 \mathrm{~h}$ after inoculation and were frozen until use; commercial kits were used for the quantification of mouse cytokines IFN- $\gamma$, IL-2, IL-4, and IL-10 (Duoset ELISA; R\&D Systems, Minneapolis, MN, USA) following the manufacturer's instructions.

\section{DETECTION OF SPECIFIC ANTIBODIES IN SERUM}

Antibodies against $\operatorname{vir} B$ mutants were quantified by indirect ELISA using a total extract of wild-type B. canis as the antigen. Three groups of 15 mice were immunized intraperitoneally with $1.4 \times 10^{8} \mathrm{CFU} / \mathrm{ml}$ of wild-type $B$. canis and its mutants, and sera were obtained at $0,7,14,21$, and 28 days post-inoculation. IgG1, IgG2a, IgG2b, and IgM isotypes were evaluated using a commercial mouse monoclonal antibody isotyping reagent kit (Sigma Aldrich, St. Louis, MO, USA) following the manufacturer's instructions. Plates were read at a wavelength of $490 \mathrm{~nm}$ (VICTOR ELISA reader).

\section{STATISTICAL ANALYSIS}

Significance levels were determined using Student's $t$-test to compare between vaccinated and unvaccinated groups and among days. A value of $P<0.05$ was considered significant.

\section{RESULTS}

\section{CHARACTERIZATION AND COMPLEMENTATION OF B. CANIS virB10::Gm AND B. CANIS $\Delta$ virB11::Gm MUTANTS}

A total of $5 \mu \mathrm{g}$ of $\mathrm{pB} 2 \mathrm{~A} 3:: \mathrm{Gm}$ plasmid was electroporated with a GenePulser (Bio-Rad) into B. canis wild-type strain; colonies displaying $\mathrm{Gm}^{\mathrm{r}}$ and $A m p^{s}$ were selected to obtain mutants with a homologous double recombination $\left(\mathrm{Gm}^{\mathrm{r}} \mathrm{Am} \mathrm{m}^{\mathrm{s}}\right)$. PCR analysis confirmed that the wild-type virB 10 gene was replaced. The mutated region was amplified to determine the interruption of the virB10 gene using primers that amplify a 1369-bp fragment 
for the parental strain and a 2069-bp fragment for the mutant strain. This procedure generated the non-polar mutant, $B$. canis virB10::Gm.

The plasmid pBlue-virB10::Gm-ORF13-ORF12 was electroporated with a GenePulser (Bio-Rad) into B. canis wild-type strain to generate the deletion of the virB11 gene. $\mathrm{Gm}^{\mathrm{r}}$ and $\mathrm{Amp}^{\mathrm{s}}$ colonies were selected and then analyzed by PCR to confirm that the replacement of virB11 with $\mathrm{Gm}$ had occurred. This procedure generated the non-polar B. canis mutant, $\Delta v \operatorname{ir} B 11:: \mathrm{Gm}$. To confirm the interruption of the virB11 gene, an amplification of the mutated region was performed using primers that amplify a 2013bp fragment for the wild-type strain and a 1900-bp fragment for the mutant strain. This procedure generated the non-polar mutant, B. canis $\Delta$ virB11::Gm.

To restore the $\operatorname{vir} B 10$ gene, a $1.2-\mathrm{kb}$ fragment containing the entire virB10 gene was amplified and cloned directly into pCR2.1 TOPO; the resulting plasmid was named pGP10c. Plasmid pGP10c was digested with EcoRI to recover a 1.2-kb fragment, which was ligated into pBBRMCS-4-EcoRI to generate a pBBR4-virB10. This plasmid was electroporated into $B$. canis virB10::Gm, and strains resistant to ampicillin were selected. The presence of the plasmid was confirmed using alkaline lysis.

To restore the virB 11 gene, the complete gene was amplified and cloned directly into pCR2.1 TOPO; the resulting plasmid was named pGP11c. This plasmid was digested with EcoRI to generate a $1.3-\mathrm{kb}$ product that contained the entire virB11 gene. The $1.3-\mathrm{kb}$ fragment was ligated into the $\mathrm{pBBR} 1 \mathrm{MCS}-4$ plasmid at the EcoRI site to generate the pBBR4-virB11 plasmid. This plasmid was transformed into $B$. canis $\Delta v i r B 11:: \mathrm{Gm}$ by electroporation, ampicillin-resistant strains were selected and the presence of the plasmid was confirmed by alkaline lysis.

\section{EXPERIMENTAL INFECTION IN MICE}

To evaluate the survival of the $\operatorname{vir} B 10$ and $\operatorname{vir} B 11$ mutants, the complemented mutants $\operatorname{vir} B 10:: \mathrm{Gm}$ and $\Delta v i r B 11:: \mathrm{Gm}$ and $B$. canis wild-type strain in vivo, groups of mice were inoculated intraperitoneally with $1.4 \times 10^{8} \mathrm{CFU}$ of the strains in $0.5 \mathrm{ml}$ of PBS; at different times, the numbers of CFU/ml were determined from spleens. Experimental results revealed that at 7 days post-infection, there were no differences in the recovered CFU of the different strains. By the third week, mutant virB10 had already been completely eliminated, thus indicating a notable reduction in virulence. Compared to the wild-type strain, mutant virB 11 reduced its bacterial load by 0.2 logs by week 3 , and at week 5 , the levels were undetectable, suggesting that the loss of some of the virB genes resulted in the elimination of the bacteria from the mouse spleens. Mutant virB11 complemented with plasmid pBBR4-virB11 recovered its virulence and replicated similarly to the wild-type strain (Figure 1).

To examine the protection induced by virB mutants, an immunization experiment was performed. Mice were inoculated i.p. with $1.4 \times 10^{8} \mathrm{CFU}$ of $\operatorname{vir} B 10:: \mathrm{Gm}, \Delta v \operatorname{ir} B 11:: \mathrm{Gm}$, or with PBS. Six weeks post-inoculation, animals were challenged with $5 \times 10^{4} \mathrm{CFU}$ of $B$. canis wild-type strain. Protection was defined as the difference between the numbers of viable bacteria recovered from spleens of immunized mice and those recovered

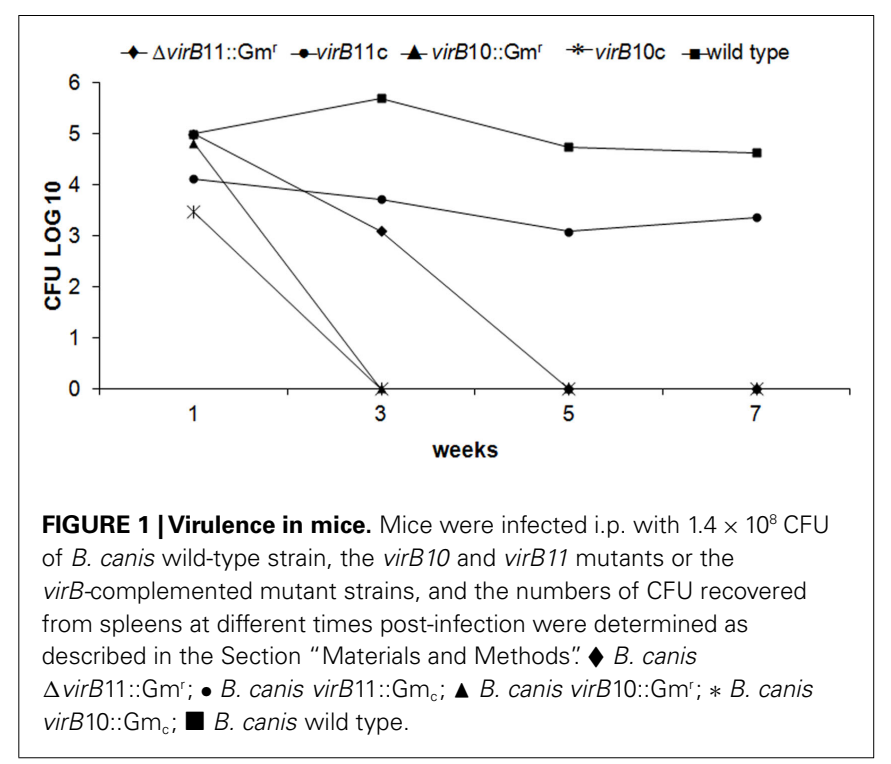

Table 1 | Protection against $B$. canis wild-type.

\begin{tabular}{|c|c|c|c|c|}
\hline \multirow[t]{2}{*}{$\begin{array}{l}\text { Treatment group } \\
(n=8) \\
\text { receiving }\end{array}$} & \multicolumn{2}{|c|}{$\begin{array}{l}\text { Mean } \log _{10} \text { of the no. of } \\
\text { brucellae } \pm \text { SD in spleens on } \\
\text { post-challenge day }\end{array}$} & \multicolumn{2}{|c|}{$\begin{array}{l}\log _{10} \text { of } \\
\text { protection } \\
\text { on day }\end{array}$} \\
\hline & 15 & 30 & 15 & 30 \\
\hline virB10::Gm & $3.84 \pm 0.58$ & $3 \pm 0.47$ & $1.72^{b}$ & $1.91^{\mathrm{b}}$ \\
\hline$\Delta$ virB11::Gm & $4.8 \pm 0.68$ & $2.95 \pm 0.46$ & 0.76 & $1.96^{b}$ \\
\hline PBS & $5.56 \pm 0.74$ & $4.91 \pm 0.69$ & & \\
\hline
\end{tabular}

${ }^{a} \log _{10}$ of protection is defined as the difference between the mean log of the numbers of CFU from immunized mice and that of the numbers of CFU from mice receiving saline. ${ }^{b} P<0.05$ (significant) compared with value for control mice.

from spleens of mice that received saline; results are summarized in Table 1. The virB10::Gm inoculation generated significant protection 15 and 30 days post-challenge (1.72 and 2 protection units, respectively). As expected, $\Delta$ virB11::Gm also induced significant protection at day 30 (1.96 protection units). These results emphasize the potential of this mutant for use as a live vaccine for dogs.

\section{MUTANTS INDUCE A HUMORAL RESPONSE SIMILAR TO WILD-TYPE INFECTION}

Sera collected at $0,7,14,21$, and 28 days post-vaccination were used to detect the presence of specific antibodies against B. canis by ELISA. Immunization with $v i r B$ mutants stimulated an antibody response with IgG1, IgG2a, IgG2b, and IgM isotypes. A similar distribution occurred in the sera of mice inoculated with the wildtype strain. Although IgG2a levels increase in mice vaccinated with $\operatorname{virB10}$, on day 21 post-infection, there are no significant differences $(P<0.05)$ compared to the sera of mice challenged with the wild-type strain, in which IgG2a levels increase up to day 28 (data not shown). 


\section{CYTOKINE RESPONSES BY virB MUTANTS}

The immune cellular response was characterized based on the secretion of cytokines at different times in supernatants of lymphocyte cultures obtained from mice previously immunized and later stimulated with the corresponding strains. The wild-type strain induces a rapid and strong INF- $\gamma$ response with a marked reduction at $96 \mathrm{~h}$, while IL-10 is slightly increased at $72 \mathrm{~h}$. There was no significant difference $(P>0.05)$ in the production of INF$\gamma$ between virB10 and virB11 mutants. IL-2 and IL-4 levels for both mutants were not statistically significant compared to those produced by the spleen cells of mice inoculated with the wild-type strain (Figure 2).

\section{DISCUSSION}

The development of a live vaccine for the control and eradication of canine brucellosis is of great importance. Nevertheless, currently, there is no information that describes an appropriate candidate that can be used as a vaccine against $B$. canis.

In this study, it was shown that $\operatorname{vir} B 10$ and $\operatorname{vir} B 11$ genes perform an essential role in $B$. canis virulence in mice. Studies published by other groups confirm these results and also show that these genes fulfill the same function in other Brucella species (Hong et al., 2000; Sieira et al., 2000; Comerci et al., 2001; Delrue et al., 2001; Boschiroli et al., 2002; Celli et al., 2003). Through the construction of mutants, it has been shown that the alteration of either virB10 or virB11 with non-polar mutations has a detrimental effect on virulence. Comerci et al. (2001) used non-polar mutations to demonstrate that $\operatorname{vir} B 4$, virB8, and $\operatorname{vir} B 10$ genes are required for the persistence of Brucella infection, while Den Hartigh et al. (2008) demonstrated that non-polar mutations in virB10 and $\operatorname{vir} B 11$ are essential for B. abortus virulence in mice.

Brucella virulence mechanisms are not fully understood, but the capacity to cause disease is related to the intracellular survival of the bacteria, which has been associated with the T4SS (Hong et al., 2000; Comerci et al., 2001; Delrue et al., 2001; Boschiroli et al., 2002; Celli et al., 2003). In this study, we conclude that the absence of $B$. canis virB 10 and virB 11 genes from the virB operon is associated with a reduction of virulence in mice. Sieira et al. (2000) demonstrated that a B. abortus virB10 mutant could not be recovered from the spleens of infected mice. In this study, non-polar virB mutants had reduced spleen counts compared to the wild-type strain, indicating severe reductions in virulence as estimated by the numbers of viable bacteria from the spleens at all times p.i that were assessed. This phenotype is likely due to a faulty secretion of effector molecules as a result of a T4SS that works deficiently in the absence of some $v i r B$ gene. We also analyzed the protection properties of $\operatorname{vir} B$ mutants in the BALB/c mouse model. Our results show that the mutants protect mice from a challenge with a virulent strain of wild-type $B$. canis with a reduction of approximately $2 \log$ units.

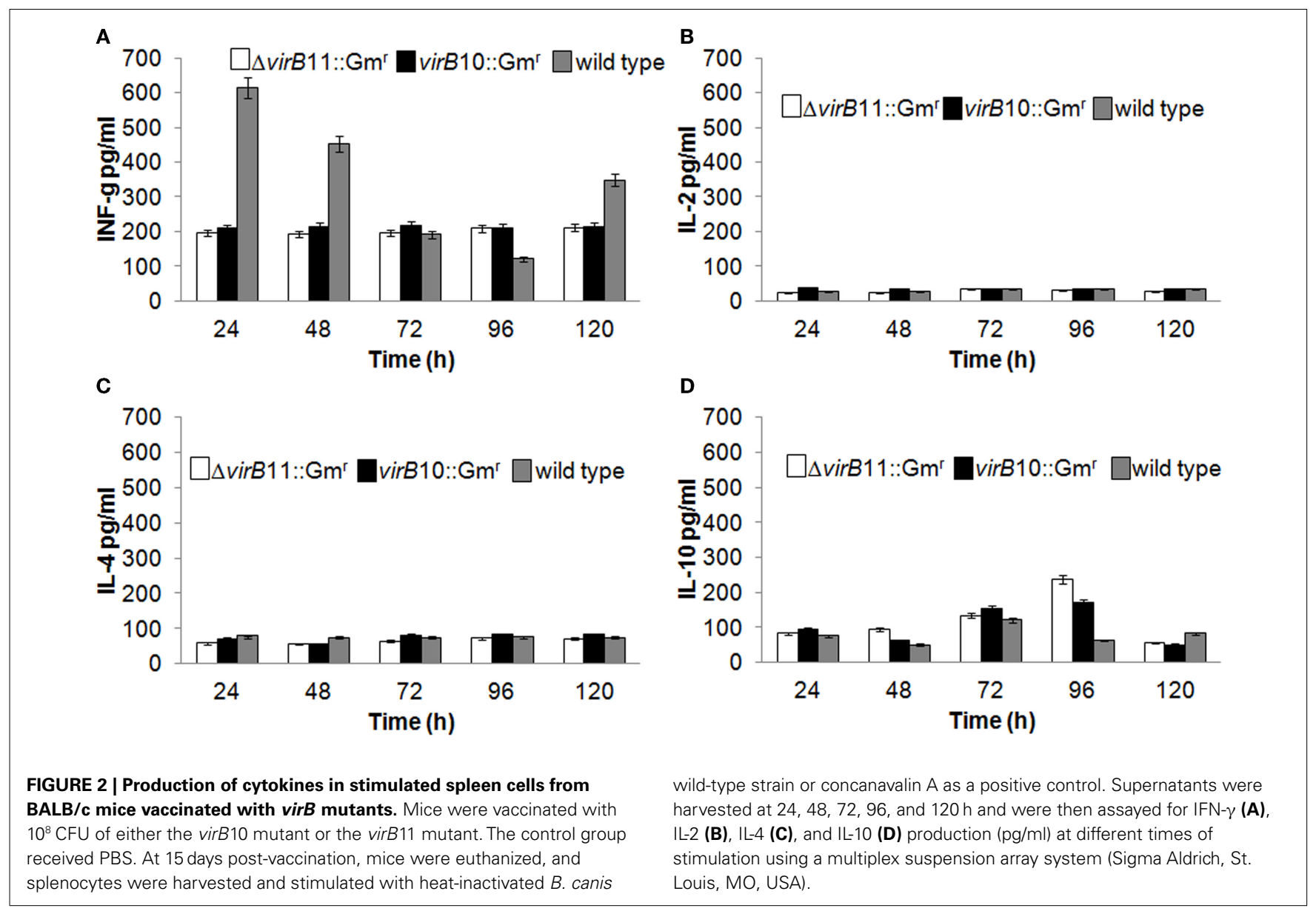


Even though there are detectable levels of anti-Brucella IgG, the antibody response to immunization was considered to be low compared to other virulence factors used in immunizations; nevertheless, the IgG1 and IgG2a levels were heterogeneous in both mutants. Our results and numerous examples in the literature indicate that INF- $\gamma$ levels produced in response to a vaccination candidate do not always correlate with the efficacy of the vaccine during a challenge with Brucella. Various in vitro studies have demonstrated that murine spleen cells previously inoculated with live vaccines induce a type Th1 response to Brucella antigens, while those immunized with inactivated vaccines induce a type Th2 response (Zhan et al., 1995). In mice infected with $B$. abortus virB mutants, serum levels of immunoglobulin IgG2a and cytokines INF- $\gamma$ and IL-12p40 increase more than in mice infected with $B$. abortus wild-type strain (Rolan

\section{REFERENCES}

Baldwin, C. L., and Goenka, R. (2006). Host immune responses to the intracellular bacteria Brucella: does the bacteria instruct the host to facilitate chronic infection? Crit. Rev. Immunol. 26, 407-442.

Baldwin, C. L., and Parent, M. (2002). Fundamentals of host immune response against Brucella abortus: what the mouse model has revealed about control of infection. Vet. Microbiol. 90, 367-382.

Boschiroli, M. L., Ouahrani-Bettache, S., Foulongne, V., MichauxCharachon, S., Bourg, G., Allardet-Servent, A., Cazevieille, C., Lavigne, J. P., Liautard, J. P., Ramuz, M., and O'Callaghan, D. (2002). Type IV secretion and Brucella virulence. Vet. Microbiol. 90, 341-348.

Celli, J., de Chastellier, C., Franchini, D. M., Pizarro-Cerda, J., Moreno, E., and Gorvel, J. P. (2003). Brucella evades macrophage killing via VirB-dependent sustained interactions with the endoplasmic reticulum. J. Exp. Med. 198, 545-556.

Comerci, D. J., Martínez-Lorenzo, M. J., Sieira, R., Gorvel, J. P., and Ugalde, R. A. (2001). Essential role of the VirB machinery in the maturation of the
Brucella abortus-containing vacuole. Cell. Microbiol. 3, 159-168.

Delrue, R. M., Martinez-Lorenzo, M., Lestrate, P., Danese, I., Bielarz, V., Mertens, P., De Bolle, X., Tibor, A., Gorvel, J. P., and Letesson, J. J. (2001). Identification of Brucella spp. genes involved in intracellular trafficking. Cell. Microbiol. 3, 487-497.

Den Hartigh, A. B., Rolan, H. G., De Hong, M. F., and Tsolis, R. M. (2008). VirB3 to VirB6 and VirB8 to VirB11, but Not VirB7, are essential for mediating persistence of Brucella in the reticuloendothelial system. $J$. Bacteriol.13, 4427-4436.

Golding, B., Scott, D. E., Scharf, O., Huang, L. Y., Zaitseva, M., Lapham, C., Eller, N., and Golding, H. (2001). Immunity and protection against Brucella abortus. Microbes Infect. 3, 43-48.

Hong, P. C., Tsolis, R. M., and Ficht, T. A. (2000). Identification of genes required for chronic persistence of Brucella abortus in mice. Infect. Immun. 68, 4102-4107.

Rolan, H. G., and Tsolis, R. M. (2008). Inactivation of the type IV secretion system reduces the Th1 polarization of the immune response to Brucella abortus infection. Infect. Immun. 76, 3207-3213.

and Tsolis, 2008). Our results suggest that the virB mutants elicit a strong protective immunity and should be considered as candidates for live vaccines for dogs. The evaluation of the mutants generated by insertion of the gentamycin resistance cassette as a selection marker is permitted in our country (Mexico); however, to be considered as a candidate for a live vaccine, it will be necessary to reconstruct the mutants and remove the gentamycin selection marker using counterselectable $s a c B$ as a selection marker.

\section{ACKNOWLEDGMENTS}

This work was supported by the Mexican "Consejo Nacional de Ciencia y Tecnología” (CONACyT), grants SEP-CONACyT (No. 58848) and TAMU-CONACyT ("Development of Brucella canis $\operatorname{virB}$ mutants, and its study in a cellular model").

Sieira, R., Comerci, D. J., Sánchez, D. O., and Ugalde, R. A. (2000). A homologue of an operon required for DNA transfer in Agrobacterium is required in Brucella abortus for virulence and intracellular multiplication. J. Bacteriol. 182, 4849-4855.

Sun, Y. H., Den Hartigh, A. B., Santos, R. L., Adams, L. G., and Tsolis, R. M. (2002). virB-Mediated survival of Brucella abortus in mice and macrophages is independent of a functional inducible nitric oxide synthase or NADPH oxidase in macrophages. Infect. Immun. 70, 4826-4832.

Sun, Y. H., Rolan, H. G., den Hartigh, A. B., Sondervan, D., and Tsolis, R. M. (2005). Brucella abortus virB12 is expressed during infection but is not an essential component of the type IV secretion system. Infect. Immun.73, 6048-6054.

Zhan, Y., Kelso, A., and Cheers, C. (1995). Differential activation of Brucella-reactive CD4+ T cells by Brucella infection or immunization with antigenic extracts. Infect. Immun. 63, 969-975.

Zhan, Y., Liu, Z., and Cheers, C. (1996). Tumor necrosis factor alpha and interleukin-12 contribute to resistance to the intracellular bacterium Brucella abortus by different mechanisms. Infect. Immun. 64 , 2782-2786.

Conflict of Interest Statement: The authors declare that the research was conducted in the absence of any commercial or financial relationships that could be construed as a potential conflict of interest.

Received: 01 August 2011; accepted: 02 March 2012; published online: 19 April 2012.

Citation: Palomares-Resendiz E, Arellano-Reynoso B, Hernández-Castro $R$, Tenorio-Gutiérrez V, Salas-Téllez E, Suárez-Güemes F and Díaz-Aparicio E (2012) Immunogenic response of Brucella canis virB10 and virB11 mutants in a murine model. Front. Cell. Inf. Microbio. 2:35. doi: 10.3389/fcimb.2012.00035 Copyright (c) 2012 Palomares-Resendiz, Arellano-Reynoso, Hernández-Castro, Tenorio-Gutiérrez, Salas-Téllez, SuárezGüemes and Díaz-Aparicio. This is an open-access article distributed under the terms of the Creative Commons Attribution Non Commercial License, which permits non-commercial use, distribution, and reproduction in other forums, provided the original authors and source are credited. 